\title{
Detection and prediction of photovoltaic panels malfunctions
}

\author{
M. Belik ${ }^{1}$ \\ ${ }^{1}$ Department of Power Engineering and Ecology \\ University of West Bohemia \\ Univerzitni 8, 30614 Pilsen (Czech Republic) \\ Phone/Fax number:+0420 376 734315, e-mail: belik4@ @ee.zcu.cz
}

\begin{abstract}
This article discusses issues of photovoltaic panels malfunctions and failures. Several computerized detection methods are applied on 300 real commercial crystalline $\mathrm{Si}$ photovoltaic installations to identify origin, quantity and time behaviour of most common malfunctions. This paper focuses only on operational failures while malfunctions derivable from fabrication process and transport are not in account. Failures influence on VA characteristic is deeply discussed. Possible usage of computerized real time VA characteristic measurements for backward failure detection and identification is evaluated. The main contribution of this research is the application and verification of this method on 8 channel pilot system.
\end{abstract}

\section{Key words}

photovoltaic panels malfunctions, detection methods, malfunction time behaviour, VA characteristic of PV panel, real time measurement.

\section{Introduction}

Photovoltaic arrays of all sizes became conventional part of power sources mix during the last decade. Although these systems contain no mechanical components they are very sensitive and vulnerable. The main disadvantage of photovoltaic systems from this point of view is installation in the open space and direct exposition to unpredictable ambient conditions. This situation can lead into wide set of operational failures or malfunctions $[1,2]$.

While the prediction of power generation from weather depending systems is always very challenging, existence of described phenomenon yet seriously obstructs this task.

Multimegawatt photovoltaic arrays and even small family house systems represent either independent energy solution or interesting economical investment. Unfortunately the real benefits often do not comply with original calculations and expectations. The reasons are evident. Pre-design computations are usually based on common simulation tools and usually tend to be rather very optimistic and do not correspond with harsh local ambient conditions. Real production is later yet often decreased through reasons of incorrectly detected and identified system malfunctions and defects $[1,2]$.

The malfunction is defined as a state when power station generates decreased or no output. Malfunctions must be detected and resolved in shortest possible time. Economical aspects of this phenomenon are evident [1].

Practical influence of particular malfunction varies in accordance of its location in the production chain as shown on Fig.1. The less serious issues can be found in the left part of the schema, while the most serious problems proceed in the right side. This statement is valid either for technical aspects or for economical point of view. Accordingly the malfunction detection can be described in the same way, but in counter direction. Serious problems in the right position on the picture are very easy to detect, while the less important malfunctions in the left area are very hard to identify and locate.

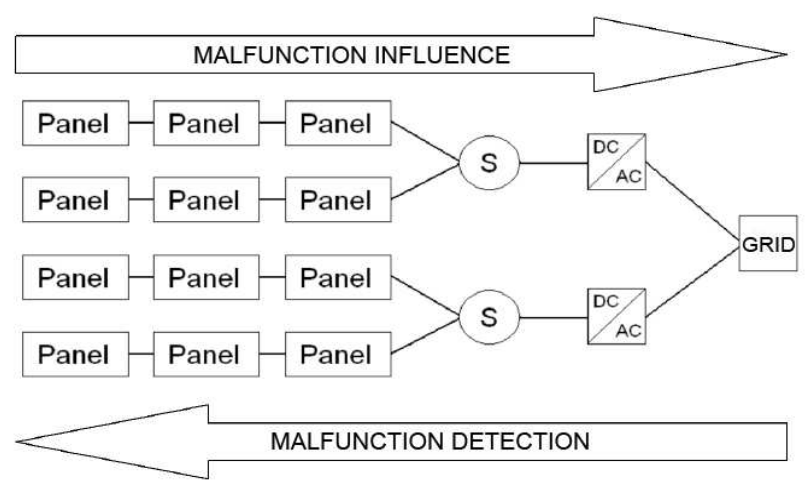

Fig. 1. Malfunction influence and detection.

Particular failures can be categorised in correspondence to their origin:

- cell malfunction

- panel malfunction

- $\quad$ string malfunction

- inverter malfunction

- construction malfunction

- $\quad$ supplementary equipment malfunction 
This classification is useful either for prevention or for identification of malfunctions. VA characteristic of real photovoltaic power station can be deformed from many reasons and the deformations look often reciprocally. If we are able to exclude some specific types of malfunctions, we are able to identify real reason of the failure with higher presumption. Shading is the typical example. We can identify areal shades of the clouds or surrounding objects or so called microshades. Although the area of the microshades seems to be rather small, their final impact tends to very serious microcracks.

Fig. 2 presents two occurrences of microshades. While the left side shows imprint of manufacturer's logo over active cell surface (result of imperfect manufacturing process), the right side demonstrates organic residues from bird's excrements as typical example of service issues. The first example can be identified and classified during the initial visual check and measurements. VA characteristic deformations typical for microshades measured within operation hours aim the second example to be quickly identified and properly solved.
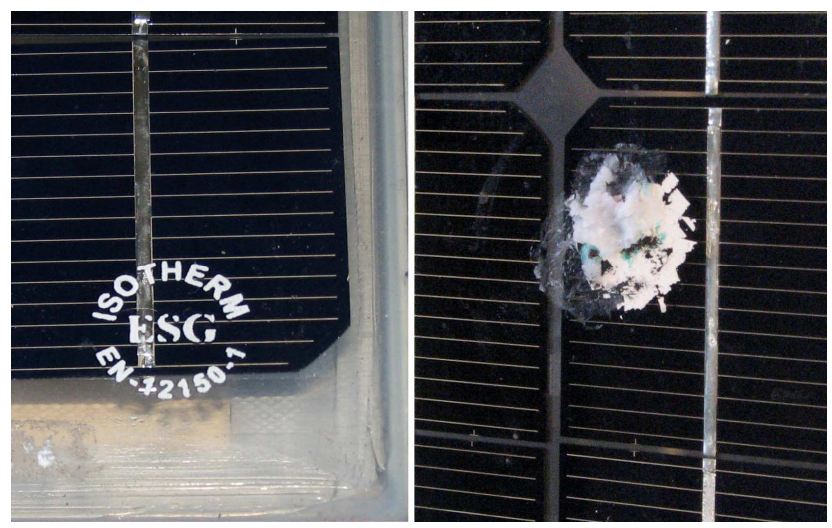

Fig. 2. Malfunction identification and classification - microshades.

Microshades are very good example of relatively small issue that is able to lead into very serious damage in close future.

\section{Operational Malfunctions Of Real PV Systems}

Question of effective frequency, importance and costs of real PV systems malfunctions initiated this research.

Operators of $300 \mathrm{PV}$ power stations with nominal output $4 \mathrm{~kW}-2 \mathrm{MW}$ from Czech republic were recipients of initial data acquisition. Regarding to used PV panel technologies, only crystalline $\mathrm{Si}$ installations were sorted out for this research. Anonymous questionnaire focused on complete set of malfunctions affecting the operation. Acquired data related to failures of inverters, constructions and supplementary equipment (protections, connection, measurement etc.) are not discussed in this article. Also basic cell malfunctions that can and should be identified during fabrication process are not mentioned [3, 9].

Table I summarizes panel and string malfunctions. Particular effects, basic grounds and recommendations for possible service intervention are explained.
Table I. - Panel and String Malfunctions List

\begin{tabular}{|l|l|l|l|}
\hline \multicolumn{1}{|c|}{ Malfunction } & \multicolumn{1}{c|}{ Effect } & \multicolumn{1}{c|}{ Origin } & \multicolumn{1}{c|}{ Solution } \\
\hline partial shading & deformed VA & external object & object remove \\
\hline delamination & lowered VA & bad material & replace \\
\hline snail traces & lowered VA & material error & replace \\
\hline low transparency & low output & material error & replace \\
\hline water in panel & high Rs & bad process & replace \\
\hline aerial pollution & low output & dust, snow... & cleaning \\
\hline local pollution & deformed VA & leafs, weeds... & cleaning \\
\hline broken glass & visual & mech. destructed & replace \\
\hline cell crack & deformed VA & mech. destructed & replace \\
\hline wrong connection & high Rs & bad process & replace \\
\hline bad connection & high Rs & corrosion & replace \\
\hline wrong bypass diode & lower output & bad process & replace \\
\hline bad bypass diode & lower output & overvoltage & replace \\
\hline cell mismatch & low output & bad process & replace \\
\hline thin wiring & high Rs & bad project & change \\
\hline wrong wiring & high Rs & bad installation & repair \\
\hline wrong connector & high Rs & bad installation & repair \\
\hline bad connector & high Rs & corrosion & repair \\
\hline modul mismatch & high Rs & bad project & reconnect \\
\hline
\end{tabular}

Totally 863 failures of various importance were analysed. Panels and strings malfunctions mean $41 \%$ while inverter malfunctions give $47 \%$, construction failures 4 $\%$ and supplementary equipment $8 \%$ from the total sum of recorded failures. Detailed overview of 353 analysed panel and string malfunctions is presented in Table II.

Table II. - Analyzed Panel and String Malfunctions

\begin{tabular}{|l|l|l|l|}
\hline \multirow{2}{*}{ Malfunction } & \multicolumn{2}{|c|}{ Suma } & \multirow{2}{*}{ Typical origin } \\
\cline { 2 - 3 } & - & $\%$ & \\
\hline partial shading & 24 & 6,8 & trees, chimney, constructions, .. \\
\hline delamination & 11 & 3,1 & material malfunction, erosion \\
\hline snail traces & 22 & 6,2 & material malfunction, microcracks \\
\hline low transparency & 5 & 1,4 & UV radiation, material malfunction \\
\hline water in panel & 6 & 1,7 & bad fabrication, mechanical stress \\
\hline aerial pollution & 46 & 13,1 & dust, mud, papers, painting \\
\hline local pollution & 89 & 25,2 & leafs, weeds, birds excrements \\
\hline broken glass & 23 & 6,5 & storm, loose stones, vandalism \\
\hline cell crack & 18 & 5,1 & mechanical stress, transportation \\
\hline wrong connection & 1 & 0,3 & human factor \\
\hline bad connection & 12 & 3,4 & corrosion, mechanical stress \\
\hline wrong bypass diode & 7 & 2,0 & bad fabrication \\
\hline bad bypass diode & 23 & 6,5 & overvoltage, overheating, lightning \\
\hline cell mismatch & 2 & 0,6 & bad fabrication \\
\hline thin wiring & 2 & 0,6 & bad project, costs savings \\
\hline wrong wiring & 5 & 1,4 & human factor \\
\hline bad wiring & 4 & 1,2 & wildlife \\
\hline wrong connector & 3 & 0,8 & human factor \\
\hline bad connector & 34 & 9,4 & corrosion, wildlife \\
\hline modul mismatch & 16 & 4,5 & costs savings \\
\hline
\end{tabular}

Listed malfunctions can be detected and classified using several methods varying from simple manual inspection through thermography to complicated and expensive special tests such as flashtest, LBIC, photoluminescence, etc. According to Table I and Table II it is evident that 93 $\%$ of analysed panel or string malfunctions affect in some way the VA characteristic $[2,3]$.

The real origin of some malfunctions can be sometimes not evident or confusing. While microcrack on Fig. 3 means damaged panel that must be replaced, panel with the same thermal behaviour on Fig. 4 is affected only with microshade originating from a bird's excrement and can be simply cleaned. 


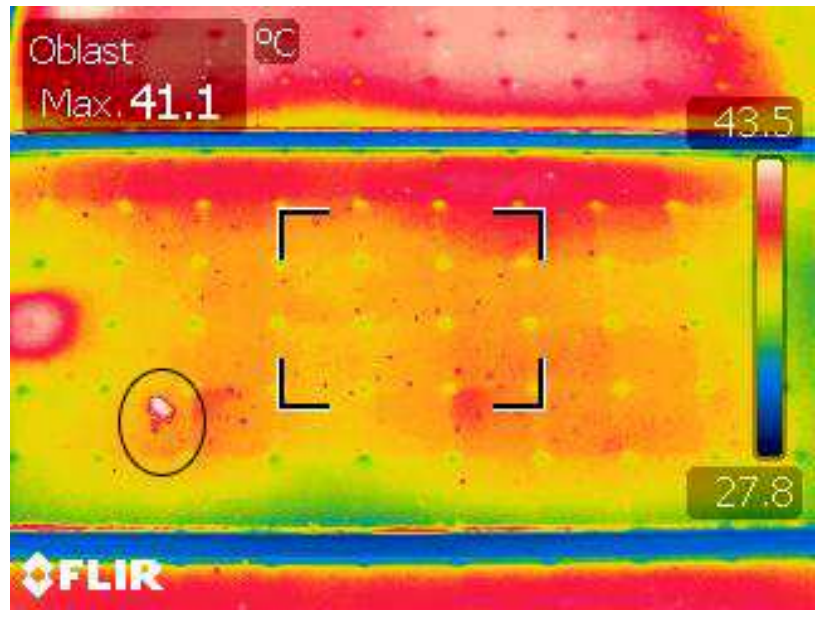

Fig. 3. Sample thermograph - microcrack.

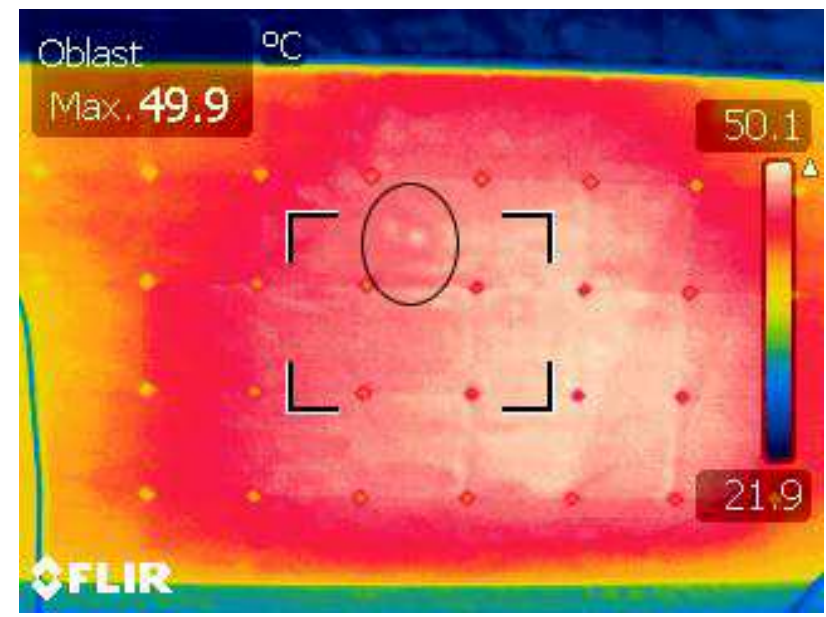

Fig. 4. Sample thermograph - microshade.

Fig. 5 shows thermograph and backside picture of a PV panel. Thermograph indicates several hotspots typical for microcracks or internal shortcuts, while the backside picture shows some smoke traces. All these phenomenons were surprisingly caused by a lightning strike into a close tree. Inducted overvoltage initiated high voltage sparks between connecting wires and backside of the panel. Correct identification of this malfunction origin is not possible without mentioned additional information. This case again demonstrates importance and involvement of failure classification [4].
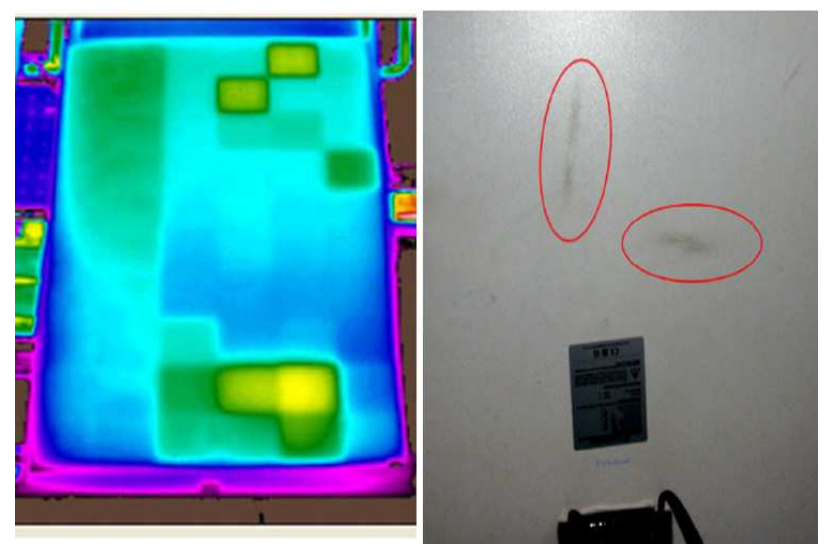

Fig. 5. Sample thermograph and picture of a panel malfunction.

\section{Failure And Malfunction Influence on VA Characteristic}

PV panel VA characteristic expressed in (1) depends either on features of the panel or on operating conditions. While the ambient conditions (radiation, temperature, spectrum) shift the curve along axes, the panel features directly affect the shape of the curve $[2,8]$.

$$
I=I_{F V}-I_{s} *\left[\exp \left(\frac{q *\left(U+I * R_{S O}\right)}{n * k * T}\right)-1\right]-\left(\frac{U+I * R_{S O}}{R_{S H}}\right)
$$

Particular values of output voltage and current depend on material features, technology of the junction, diffusion and layers deposition. The slope of the curve is affected by serial and parallel resistance and corresponds with material purity and quality of particular manufacturing stages. Fig. 6 illustrates sample VA and power characteristic of a non-defective PV panel. As discussed earlier, several parameters have often similar final effect. Clear identification of particular phenomenon is not possible from single general measurement without additional information set $[4,5,6]$.

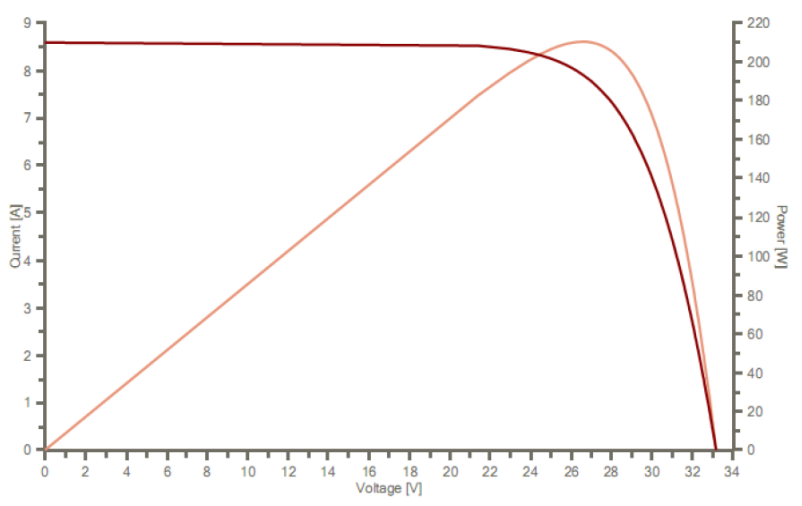

Fig. 6. VA and power characteristic of non-defective PV panel.

In contrast of that, continuous shape changes of the VA characteristic can be used for identification and primarily for detection of malfunctions. The only condition for practical usage of this method is application of sufficient measurement and analysing system. The analysis is based on history of previously recorded measurements. The period depends on demanding accuracy. The shorter is the interval, the smaller difference and smaller malfunction can be detected.

\section{Online VA Characteristic Measurement}

To apply presented ideas, sufficient realtime data are required. Realtime measurement and analysis of VA characteristics is rather complicated and interferes with power production. Correct measurement requires defined load. It means that analysed system must be temporarily disconnected from its own load (typically inverter) and connected to variable retuning load of the analyser. While the VA characteristic can and must be measurement fast, switching operations can be easily executed with controlled contactor. The triggering can be either cyclic or can depend on actual state of the PV system. 
Fixed interval contactor switching in predefined times is easy to build but is not capable to detect unfavourable system behaviour between these points. The other option is to measure VA characteristic "on request". It originates from detected unusual differences between realtime measured operating parameters.

Hardware of developed experimental instrumentation system is based on data acquisition device National Instruments NI-PCI 6221. 16 analogue channels of this device allow simultaneous measurement of 8 separate PV panels. One channel is used for voltage and one for indirect current measurements [7].

Usage of another additional channel for each panel enables simultaneous measurement of actual panel temperature. One separate channel is used for measurement of solar radiation (identical for all panels). This advanced option increases the sensitivity but decreases number of parallel panels to five.

16 bit bus and fast sampling frequency enable short intervals and thus detection of small differences. Voltage range $\pm 10 \mathrm{~V}$ requires coupling resistances as shown on Fig. 7 and allows adaptation for wide range of PV panels. Current sampler with transducer is complementary wiring option.

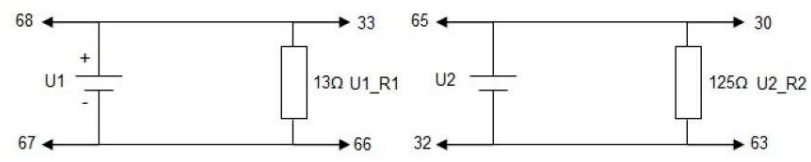

Fig. 7. Connection of coupling resistances to DAQ connector block.

Measured data are recorded and analysed through developed service system based on LabVIEW environment.

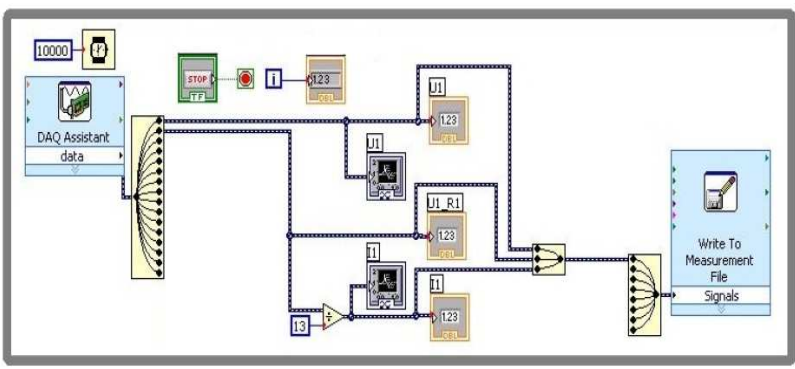

Fig. 8. Block diagram for 1 PV Panel.

Wire diagram of the basic measurement 2 channel block for $1 \mathrm{PV}$ panel is displayed on Fig. 8. Final detection routine consists from 8 identical blocks coupled in 2 stages. Difference value between the stages is used for triggering of VA characteristic measurement. New characteristic is compared to stored set of previous measurements. Appropriate detected significant difference starts predefined alarm action. Punctual rate of the difference value must be set empirically for each system at the beginning. Usage of 3 channel blocks decreases the number of requested VA characteristic measurements and thus number of contactor switching operations and the influence on power production. Supplementary values of panel temperature and incident solar radiation perform as additional filter for detection of VA characteristic differences.

\section{Failure Detection Based On VA Characteristic}

Overall 37 model situations simulating different types and scales of typical operating malfunctions were used for evaluation of described method on PV panel Volther PowerVolt 190/500. The panel consists from 72 mono-Si cells wired into 3 strings. Each string is equipped with bypass diode.

Measurements 1 - 6 simulated failure-free operation and were used for system calibration. Measurements $7-16$ simulated set of defective $1-36$ cells in particular configurations while measurements $17-21$ demonstrated influence of local microshades $(0,35-1,39 \%)$. The other measurements simulated partial shading of different configuration and intensity $(2,78-11,11 \%)$.

Table III presents sample of measurements $7-16$ (defective cells). Intensity of solar radiation I varies between 731 and $777 \mathrm{~W} . \mathrm{m}^{-2}$ and temperature T between 14,9 and $21,1^{\circ} \mathrm{C}$. Because the ambient conditions are analogous, this sample set can be used for the VA characteristics comparison presented on Fig. 9.

Table III. - Sample of Measurements 7 - 16 (defective cells)

\begin{tabular}{|c|c|c|c|c|c|c|c|c|}
\hline Measurement & $\mathrm{P}_{\mathrm{m}}[\mathrm{W}]$ & $\mathrm{U}_{\mathrm{oc}}[\mathrm{V}]$ & $\mathrm{U}_{\mathrm{m}}[\mathrm{V}]$ & $\mathrm{I}_{\mathrm{m}}[\mathrm{A}]$ & $\mathrm{I}_{\mathrm{sc}}[\mathrm{A}]$ & $\mathrm{I}\left[\mathrm{W} \cdot \mathrm{m}^{-2}\right]$ & $\mathrm{T}\left[{ }^{\circ} \mathrm{C}\right]$ & Fill Factor $[\%]$ \\
\hline 7 & 142,98 & 38,96 & 29,44 & 4,86 & 5,34 & 731,00 & 17,40 & 69,00 \\
\hline 8 & 95,15 & 38,87 & 19,22 & 4,95 & 5,46 & 749,00 & 21,10 & 45,00 \\
\hline 9 & 96,10 & 38,77 & 19,44 & 4,94 & 5,50 & 754,00 & 18,40 & 45,00 \\
\hline 10 & 44,93 & 38,53 & 9,22 & 4,87 & 5,39 & 755,00 & 18,90 & 22,00 \\
\hline 11 & 45,31 & 38,59 & 9,00 & 5,03 & 5,37 & 759,00 & 19,80 & 22,00 \\
\hline 12 & 2,03 & 38,41 & 33,80 & 0,06 & 0,14 & 757,00 & 17,20 & 39,00 \\
\hline 13 & 1,89 & 38,73 & 22,23 & 0,09 & 0,15 & 777,00 & 14,90 & 34,00 \\
\hline 14 & 98,00 & 37,86 & 19,58 & 5,01 & 5,55 & 771,00 & 16,20 & 47,00 \\
\hline 15 & 98,08 & 33,21 & 19,22 & 5,10 & 5,62 & 770,00 & 16,80 & 53,00 \\
\hline 16 & 46,22 & 29,16 & 9,08 & 5,09 & 5,52 & 771,00 & 17,60 & 29,00 \\
\hline
\end{tabular}

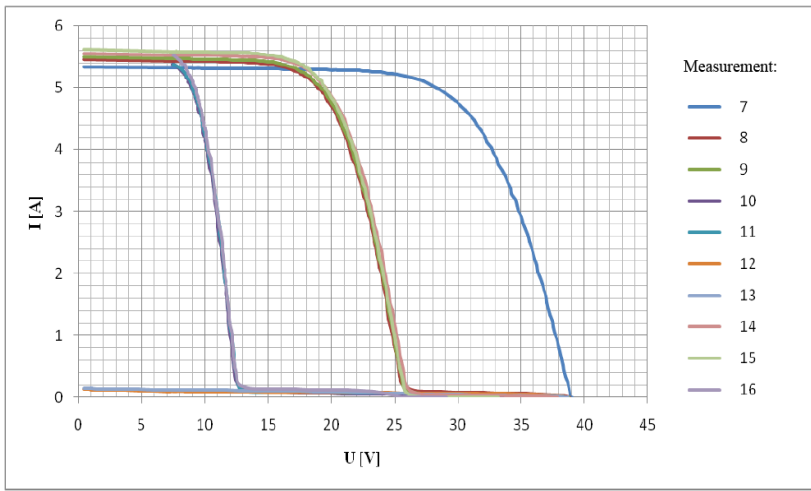

Fig. 9. VA characteristic - measurements 7 - 16 (defective cells).

Configuration of defective cells:

No. 7 - no defective cell

No. 8 - 1 defective cell

No. 9 - 2 defective cells in 1 row

No. 10 - 3 defective cells in 1 row

No. 11 - 4 defective cells in 1 row

No. 12 - 5 defective cells in 1 row

No. 13 - 6 defective cells in 1 row 
No. 14 - 12 defective cells in 1 column

No. $15-24$ defective cells in 2 columns

No. 16 - 36 defective cells in 3 columns

Table IV shows sample of measurements $17-21$ (microshades). The intensity of solar radiation I varies between 731 and $749 \mathrm{~W} . \mathrm{m}^{-2}$ and temperature T between 17,4 and $21,1^{\circ} \mathrm{C}$, so that this sample can be also used for the VA characteristics comparison as displayed on Fig. 10.

Table IV. - Sample of Measurements 17 - 21 (microshades)

\begin{tabular}{|c|c|c|c|c|c|c|c|c|}
\hline Measurement & $\mathrm{P}_{\mathrm{m}}[\mathrm{W}]$ & $\mathrm{U}_{\mathrm{oc}}[\mathrm{V}]$ & $\mathrm{U}_{\mathrm{mI}}[\mathrm{V}]$ & $\mathrm{I}_{\mathrm{m}}[\mathrm{A}]$ & $\mathrm{I}_{\mathrm{sc}}[\mathrm{A}]$ & $\mathrm{I}\left[\mathrm{W} \cdot \mathrm{m}^{-2}\right]$ & $\mathrm{T}\left[{ }^{\circ} \mathrm{C}\right]$ & Fill Factor [\%] \\
\hline 17 & 142,98 & 38,96 & 29,44 & 4,86 & 5,34 & 731,00 & 17,40 & 69,00 \\
\hline 18 & 125,31 & 39,10 & 33,23 & 3,77 & 5,41 & 739,00 & 17,90 & 59,00 \\
\hline 19 & 94,99 & 39,09 & 19,30 & 4,92 & 5,41 & 740,00 & 18,50 & 45,00 \\
\hline 20 & 94,86 & 39,01 & 19,15 & 4,95 & 5,42 & 745,00 & 19,40 & 45,00 \\
\hline 21 & 95,15 & 38,87 & 19,22 & 4,95 & 5,46 & 749,00 & 21,10 & 45,00 \\
\hline
\end{tabular}

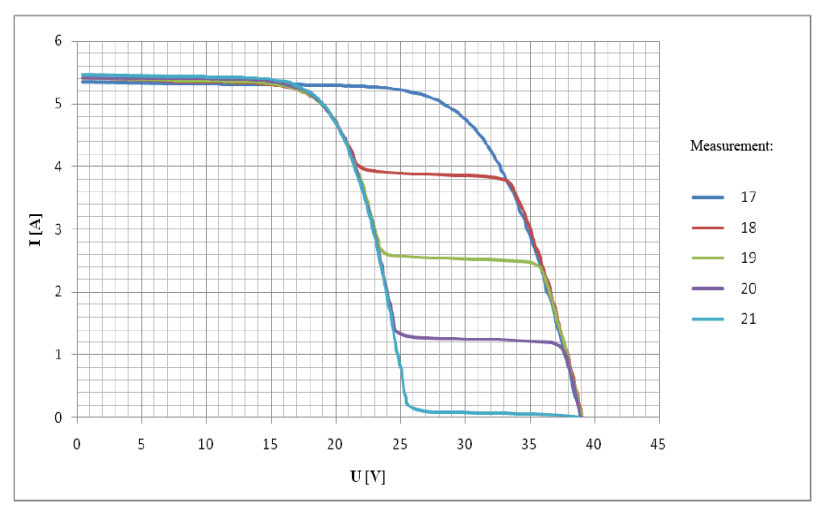

Fig. 10. VA characteristic - measurements $7-16$ (microshades).

Configuration of microshades:

No. 17 - no microshades

No. $18-1 / 4$ of 1 cell $(0,35 \%)$

No. $19-1 / 2$ of 1 cell $(0,69 \%)$

No. $20-3 / 4$ of 1 cell $(1,04 \%)$

No. $21-4 / 4$ of 1 cell $(1,39 \%)$

Sample of measurements $24-26$ (shading) is summarized in Table V. Values of solar radiation intensity I oscillate between 731 and 786 W.m ${ }^{-2}$ and temperature T between 17,4 and $19,4^{\circ} \mathrm{C}$, so this sample can be also used for the VA characteristics comparison. The results are presented on Fig. 11.

Table V. - Sample of Measurements 24 - 26 (shading)

\begin{tabular}{|c|c|c|c|c|c|c|c|c|}
\hline Measurement & $\mathrm{P}_{\mathrm{m}}[\mathrm{W}]$ & $\mathrm{U}_{\mathrm{oc}}[\mathrm{V}]$ & $\mathrm{U}_{\mathrm{m}}[\mathrm{V}]$ & $\mathrm{I}_{\mathrm{m}}[\mathrm{A}]$ & $\mathrm{I}_{\mathrm{sc}}[\mathrm{A}]$ & $\mathrm{I}\left[\mathrm{W} \mathrm{m}^{-2}\right]$ & $\mathrm{T}[\mathrm{C}]$ & Fill Factor [\%] \\
\hline 24 & 142,98 & 38,96 & 29,44 & 4,86 & 5,34 & 731,00 & 17,40 & 69,00 \\
\hline 25 & 132,97 & 39,10 & 32,44 & 4,10 & 5,61 & 786,00 & 19,00 & 61,00 \\
\hline 26 & 94,86 & 39,01 & 19,15 & 4,95 & 5,42 & 745,00 & 19,40 & 45,00 \\
\hline
\end{tabular}

Configuration of shaded cells:

No. 24 - no shading

No. $25-3 \times 1 / 4$ of 1 cell $(1,04 \%)$

No. $26-3 / 4$ of 1 single cell $(1,04 \%)$

Amount of 16 different shadings was analysed during this measurements. Shade intensity varied from $0 \%$ to $75 \%$. Model shades covered from $0 \%$ to $11,11 \%$ of the panel with different geometry (vertical or horizontal orientation).

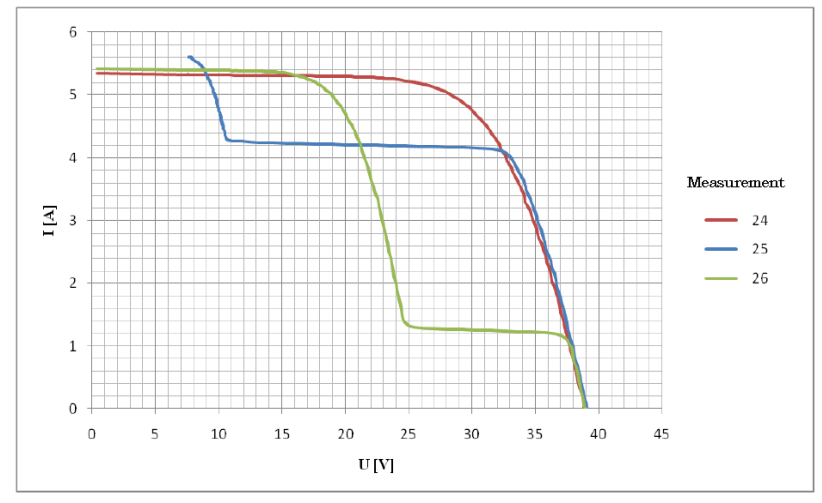

Fig. 11. VA characteristic - measurements $24-26$ (shading).

\section{Results and Conclusions}

Sample measurements presented in chapter 5 have proven early hypothetical expectations. Different types of operating malfunctions have characteristic influence on shape of the VA characteristic. Typical deformations for particular malfunctions in the sample set are visualized on Fig. 9 - Fig. 11.

Developed automated acquisition system is based on LabVIEW application and is capable for realtime measurements of PV system. This DAQ system can be used for detection and identification of operating malfunctions.

Detection consists from 2 stage measurement based on balancing of measured operating values (output voltage, output current, panel temperature and incident solar radiation). If some unexpected $\mathrm{PV}$ system behaviour is detected, controlled contactor switches VA characteristic measurement. Measured VA characteristic is compared to stored history and proves or disproves the initial detection. If some malfunction is detected, shape of the curve identifies the origin.

Future research should focus on more complex numerical analysis of measured VA characteristics to develop more general application for larger systems consisting from larger number of either uniform or non-uniform panels.

\section{References}

[1] Chu, Y. and Meisen, P., Review and Comparison of Different Solar Energy Technologies. Report of Global Energy Network Institute (GENI), Diego. 2011.

[2] Fahrenbruch, A. L., Bube, R. H.,Fundamentals of Solar Cells. Academic Press Inc., New York. 2016.

[3] Salinger, J., Benda, V., Machacek, Z., A note on solar cell diagnostics using LBIC and LBIV methods. Microelectronics, 2008. MIEL 2008.

[4] Belik, M., PV panels under lightning conditions. Electric Power Engineering (EPE), 2014.

[5] Cerna, L., A Simple Method of Evaluating Thermograms of Photovoltaic Modules. Proceedings 31st European Photovoltaic Solar Energy Conference and Exhibition. Hamburg, 2015.

[6] Cerna, L., Diagnostic of photovoltaic modules using alternating methods of measurement, Proceedings 8. CFVK, Brno, 2013

[7] Riha, T., Instrumentation system for photovoltaic applications, Diploma thesis, ZCU, Plzen, 2011.

[8] M. Libra, V. Poulek, Solarni energie, CZU, Praha, 2005.

[9] Baxa, M., Failure detection in PV systems, Diploma thesis., ZCU, Plzen, 2010. 